\title{
The use of monopole and dipole sources in crosswell surveying
}

\author{
Julieta Antonio*, António Tadeu \\ Department of Civil Engineering, University of Coimbra, Polo II-Pinhal de Marrocos, P-3030-290 Coimbra, Portugal
}

Received 30 November 2001; accepted 5 July 2004

\begin{abstract}
This paper implements a boundary element method (BEM) solution, formulated in the frequency domain, to simulate the crosswell S wave surveying technique. In this technique, one fluid-filled borehole hosts the source, and the other the receivers. The system is excited by a monopole or a dipole source placed near the first wall of the borehole wall, while the pressure field is recorded in the second borehole. The three-dimensional solution is computed as a summation of 2.5D solutions for different axial wave numbers. This model is used to assess the influence of the distance between boreholes and the material properties of the medium on the pressure field generated in the second borehole. Slow and fast formations are both simulated. It was found that the responses recorded the contribution of the non-dispersive body waves (the dilatational (P) and shear (S) waves) as well as the effect of dispersive waves associated with different wave modes. The final time solutions are thus intricate, exhibiting wave patterns that may make it difficult to interpret the arrival times of the refracted $\mathrm{P}$ and $\mathrm{S}$ waves.
\end{abstract}

(C) 2004 Elsevier B.V. All rights reserved.

Keywords: Wave propagation; Monopole and dipole sources; Crosswell surveying

\section{Introduction}

Measurement of the pressure inside a fluid-filled borehole, generated by a source either on the ground surface or in another borehole, is an essential part of several geophysical and seismic prospecting techniques (Albright and Johnson, 1990; Krohn, 1992; Toksöz et al., 1992). The

* Corresponding author. Tel.: +351 239 797196; fax: +351 239 797190.

E-mail address: julieta@dec.uc.pt (J. Antonio). interpretation of the signals recorded during the course of certain seismic testing techniques requires a full understanding of how the waves propagate from the source to the receiver. The complexity of the wave patterns recorded at the receivers depends on the relative participation of the many wave propagation modes that may be excited by the source. This contribution is a function of the distance from the source, the dominant frequency of the pulse, the material characteristics of the formation, the position of the tool relative to the axis of the borehole, the existence of casing, and the distance between boreholes (in the case of 
crosswell seismic surveys). The ratio between the shear velocity $\beta$ of the solid medium and the dilatational wave velocity of the fluid $\alpha_{\mathrm{f}}$ defines two distinct behaviors for wave propagation - when $\beta>\alpha_{\mathrm{f}}$ the medium is said to be a fast formation; otherwise, it is called a slow formation.

Different numerical methods have been used to obtain the solution of wave propagation across and within fluid-filled boreholes. The finite difference method (Stephen et al., 1985; Randall, 1991; Leslie and Randall, 1992; Yoon and McMechan, 1992; Cheng et al., 1995; Peng and Toksöz, 1995), the boundary integral approach (Bouchon and Schmitt, 1989), the boundary element method (Bouchon, 1993; Dong et al., 1995) and hybrid methods (White and Sengbush, 1963; Ben-Menahem and Kostek, 1990; De Hoop et al., 1994) are among the techniques most often used.

Peng et al. (1996) made use of both the borehole coupling theory and the global matrix formulation for computing synthetic seismograms in a layered medium. The global matrix formulation is used to calculate the stress field at the borehole location. Borehole coupling theory is then employed to obtain the pressure in the borehole fluid. No discretization along the borehole is required in this model, and the method gives results for open, cased and partially filled boreholes. The influence of the casing on the propagation of waves along fluid filled boreholes was also studied by Gibson and Peng (1994), Winbow (1991), Peng et al. (1994), and Gibson (1994).
The boundary element method (BEM) is a suitable tool for analyzing wave propagation in the vicinity of a borehole in a homogeneous isotropic formation, because it automatically satisfies the far-field conditions. The method was used by Bouchon (1993) in an infinitely long borehole placed in layered isotropic media. Dong et al. (1995) broadened the scope of Bouchon's work by incorporating transversely isotropic layers and by including the effect of casing and cement in the formation. Their work used an indirect boundary element method to model source radiation from open and cased boreholes in layered, transversely isotropic media.

This work uses the BEM to model the crosswell seismic prospecting technique, using two fluidfilled boreholes. One of the boreholes hosts a monopole or a dipole source, while the receivers are placed in the second borehole. The BEM model has been implemented and developed without any simplification, taking into account the full coupling between the solid and the fluid. The methodology extends the analyses previously performed by the authors (Tadeu et al., 2002). The methodology has been validated by solving the case of a single borehole, for which analytical solutions are known.

In the next section, the problem is defined and the BEM solutions in the frequency domain are given. Then, the BEM models are used to assess how the distance between the two boreholes and the elastic formation properties influences the propagation of different wave modes.

\section{Problem formulation}

An unbounded homogeneous isotropic elastic medium, without intrinsic attenuation, with density $\rho$, where a shear wave and a compressional wave propagate with velocity $\beta$ and $\alpha$, respectively, hosts two boreholes. A Cartesian coordinate system is centered on the axis of the borehole hosting the source, with the $z$-axis being aligned along its axis. The boreholes are assumed to be filled with an inviscid fluid with density $\rho_{\mathrm{f}}$, where the compressional waves propagate with a velocity of $\alpha_{\mathrm{f}}$. A dilatational point source, placed in one of the boreholes at position $x_{0}, y_{0}, z_{0}$, oscillating with a frequency $\omega$, disturbs the fluid, emitting an incident field that can be expressed by the dilatational potential $\phi$,

$$
\phi_{\text {inc }}=\left(-\frac{\alpha_{\mathrm{f}}^{2}}{\omega^{2} \lambda_{\mathrm{f}}}\right) \frac{A e^{i \frac{\omega}{\alpha_{\mathrm{f}}}\left(\alpha_{\mathrm{f}} t-\sqrt{\left(x-x_{0}\right)^{2}+\left(y-y_{0}\right)^{2}\left(z-z_{0}\right)^{2}}\right)}}{\sqrt{\left(x-x_{0}\right)^{2}+\left(y-y_{0}\right)^{2}\left(z-z_{0}\right)^{2}}}
$$

where $A$ is the amplitude and $\lambda_{\mathrm{f}}$ is the fluid Lamé constant. 
As the geometry of the problem does not vary along the $z$ direction, the three-dimensional solution is commonly computed as a summation of two-dimensional problems, following the Fourier transformation of the problem in the $z$ direction. This summation is rendered discrete if there is a set of virtual sources, equally spaced along the $z$ direction, (Bouchon and Aki, 1977). The resulting discrete two-dimensional problems are solved for a varying sequence of radial wave numbers $k_{\alpha \mathrm{f}}=\sqrt{\frac{\omega^{2}}{\alpha_{\mathrm{f}}^{2}}-k_{z m}^{2}}$, with $\operatorname{Im} k_{\alpha \mathrm{f}}<0$, where $k_{z m}=\frac{2 \pi}{L} m$ is the axial (longitudinal) wave number $(m=0,-1,+1 \ldots)$, and $L$ is the distance between virtual point sources equally spaced along $z$. The incident field is expressed by the potential

$$
\hat{\phi}_{\text {inc }}\left(\omega, x, y, k_{z}\right)=\left(-\frac{\alpha_{\mathrm{f}}^{2}}{\omega^{2} \lambda_{\mathrm{f}}}\right) \frac{-i A}{2} H_{0}^{(2)}\left(k_{\alpha \mathrm{f}} \sqrt{\left(x-x_{0}\right)^{2}+\left(y-y_{0}\right)^{2}}\right)
$$

in which $H_{n}^{(2)}(\ldots)$ are second Hankel functions of order $n$. The distance $L$ must be sufficiently large to avoid the contribution of the virtual sources to the response. The $2.5 \mathrm{D}$ incident pressure field is given by $\frac{-i A}{2} H_{0}^{(2)}\left(k_{\alpha \mathrm{f}} \sqrt{\left(x-x_{0}\right)^{2}+\left(y-y_{0}\right)^{2}}\right)$.

The BEM is ideally suited to solve this problem, since it automatically takes into account the far-field conditions, requiring only the discretization of the boundary of the two boreholes. The BEM equations needed to solve this problem are well-known (Beskos, 1997; Tadeu et al., 2002) and are thus not fully described here. The authors have used a similar technique for evaluating the three-dimensional wavefield elicited by monopole sources in the vicinity of fluid-filled irregular boreholes (Tadeu and Santos, 2001).

It is enough to state that the application of the method in the frequency domain requires the evaluation of the integrals along the appropriately discretized boundary of the borehole

$$
\begin{aligned}
H_{i j}^{(s) k l}=\int_{C_{l}} H_{i j}^{(s)}\left(x_{k}, x_{l}, n_{l}\right) d C_{l} & (i, j=1,2,3) & H_{f 1}^{(f) k l}=\int_{C_{l}} H_{f 1}^{(f)}\left(x_{k}, x_{l}, n_{l}\right) d C_{l} \\
G_{i j}^{(s) k l}=\int_{C_{l}} G_{i j}^{(s)}\left(x_{k}, x_{l}\right) d C_{l} & (i=1,2,3 ; j=1) & G_{f 1}^{(f) k l}=\int_{C_{l}} G_{f 1}^{(f)}\left(x_{k}, x_{l}\right) d C_{l}
\end{aligned}
$$

In these equations, $H_{i j}^{(s)}\left(x_{k}, x_{l}, n_{l}\right)$ and $G_{i j}^{(s)}\left(x_{k}, x_{l}\right)$ are, respectively, the Green's tensor for traction and displacement components in the elastic medium, at the point $x_{l}$ in direction $j$, originated by a concentrated load acting at the source point $x_{k}$ in direction $i ; H_{f 1}^{(f)}\left(x_{k}, x_{l}, n_{l}\right)$ are the components of the Green's tensor for pressure in the fluid medium, at the point $x_{l}$, due to a pressure load placed at the source point $x_{k} ; G_{f 1}^{(f)}\left(x_{k}, x_{l}\right)$ are the components of the Green's tensor for displacement in the fluid medium, at point $x_{l}$ in the normal direction, caused by a pressure load acting at the source point $x_{k} ; n_{l}$ is the unit outward normal for the $l$ th boundary segment $C_{l}$; the subscripts $i, j=1,2,3$ refer to the normal, tangential and $z$ directions, respectively. These equations are appropriately transformed from the Cartesian coordinate system $(x, y, z)$ to the tangential and normal directions of the boundary element, using standard vector transformation operators.

The required two-and-a-half dimensional fundamental solution (Green's functions) and stress functions in Cartesian coordinates, for the elastic and fluid media, are described in Tadeu and Kausel (2000).

The boundary conditions prescribed at the solid-fluid interface are the continuity of normal displacements and stresses, and null tangential stresses. The imposition of these conditions leads to a system of equations that can be solved for the nodal solid displacements and fluid pressures. The required integrations in Eq. (3) are achieved using Gaussian quadrature when the element to be integrated is not the loaded element. For the loaded element, the existing singular integrals are carried out in closed form (Tadeu et al., 1999a,b).

Solutions in the time domain are computed after the response in the frequency domain has been obtained. The required Fourier transformations are carried out by discrete summations over wave numbers and frequencies, which is equivalent to adding periodic sources at spatial intervals $L=2 \pi / \Delta k_{z}$ (on the $z$-axis), and temporal intervals 
$T=2 \pi / \Delta \omega$, where $\Delta k_{z}$ and $\Delta \omega$ are the wave number and frequency increments, respectively (Bouchon and Aki, 1977). As mentioned above, the spatial separation $L$ must be large enough to prevent contamination of the response by the periodic sources. In other words, the response given by the fictitious sources must appear at times later than $T$. Complex frequencies with a small imaginary part of the form $\omega_{c}=\omega-i \eta$ are used, mainly to avoid the aliasing phenomena. We chose $\eta=0.7 \Delta \omega$ as the imaginary part of the angular frequency, to attenuate the wraparound by a factor of $e^{0.7 \Delta \omega T}$, i.e., $38 \mathrm{~dB}$. This technique also leads to a significant attenuation, or even the virtual elimination, of the periodic sources. In the time domain, this procedure is taken into account by applying an exponential window $e^{\eta t}$ to the response (Kausel, 1992).

Solutions in the time domain are computed by modeling a source with a time evolution given by a Ricker pulse.

\section{Validation of the BEM algorithm}

The BEM code was validated by solving the case of a cylindrical circular cavity (radius $0.1016 \mathrm{~m}$ ), filled with an inviscid fluid and placed in a fast formation with properties as listed in Table 1.

This system is subjected to a spatial harmonic line source, $k_{z}=2.0 \mathrm{rad} / \mathrm{m}$, applied to the axis of the borehole. In this case, the same fluid-filled circular borehole hosts the source and the receivers. The geometry of the borehole is regular, and this allows the solution to be obtained in closed form. These solutions are well-known and can be found in the literature (see Pao and Mow, 1973 and Tadeu et al., 2001).

The response is calculated at a receiver placed in the fluid medium close to the borehole wall (see Fig. 1a). Computations are performed in the frequency range 40.0-20480.0 Hz.

In the BEM code, the number of elements varies with the frequency, assuming the ratio of the wavelength of the incident waves to the length of the boundary elements to be at least 28.0. However, a minimum of 120 boundary elements is used to discretize the inclusion.

Fig. $1 \mathrm{~b}$ displays the scattered field calculated when the borehole is modeled with constant boundary

Table 1

Formation properties

\begin{tabular}{llll}
\hline & $\begin{array}{l}\text { Compressional wave } \\
\text { velocity }(\mathrm{m} / \mathrm{s})\end{array}$ & $\begin{array}{l}\text { Shear wave } \\
\text { velocity }(\mathrm{m} / \mathrm{s})\end{array}$ & $\begin{array}{l}\text { Density } \\
\left(\mathrm{kg} / \mathrm{m}^{3}\right)\end{array}$ \\
\hline Fast formation & $\alpha=4208$ & $\beta=2656$ & $\rho=2140$ \\
Slow formation & $\alpha=2630$ & $\beta=1416$ & $\rho=2250$ \\
Fluid & $\alpha_{\mathrm{f}}=1500$ & - & $\rho f=1000$ \\
\hline
\end{tabular}

elements. Analysis of the results confirms a good agreement between the two solutions.

\section{Numerical applications}

Different crosswell $\mathrm{S}$ wave simulations are performed to understand how the distance between the two boreholes and the elastic formation properties influence the propagation of different wave types. Dipole and monopole sources are used. The dipole source is built up from a combination of two monopole sources of opposite sign and weight, 1/2, placed close together in the same horizontal plane (Kurkjian and Shu-Kong, 1986), as shown in Fig. 2. The distance between the monopole sources is set to $\delta=0.01 \mathrm{~m}$. The sources are close to the borehole wall (Fig. 3a and b). Two lines of 601 receivers are equally spaced $(0.05 \mathrm{~m})$ along the $z$ direction, from $z=0.0$ to $z=30.0 \mathrm{~m}$, one on the axis of the borehole (receiver line R1), and the other close to the borehole wall (receiver line R2), which is placed in the same azimuth direction as the source (see Fig. 3c). The plane $z=0.0 \mathrm{~m}$ passes through the source. The geometry of the crosswell model, including the source location, the receiver locations, the distances between the wells and the orientation of the coordinate system is illustrated in Fig. 3d.

The examples provided in this work consider fluid-filled boreholes (radius $0.1016 \mathrm{~m}$ ) placed inside a fast or slow formation, as studied by Ellefsen (1990), with the properties listed in Table 1. Frequency and time results are displayed. The results obtained with a fast formation are analyzed next. 
a)
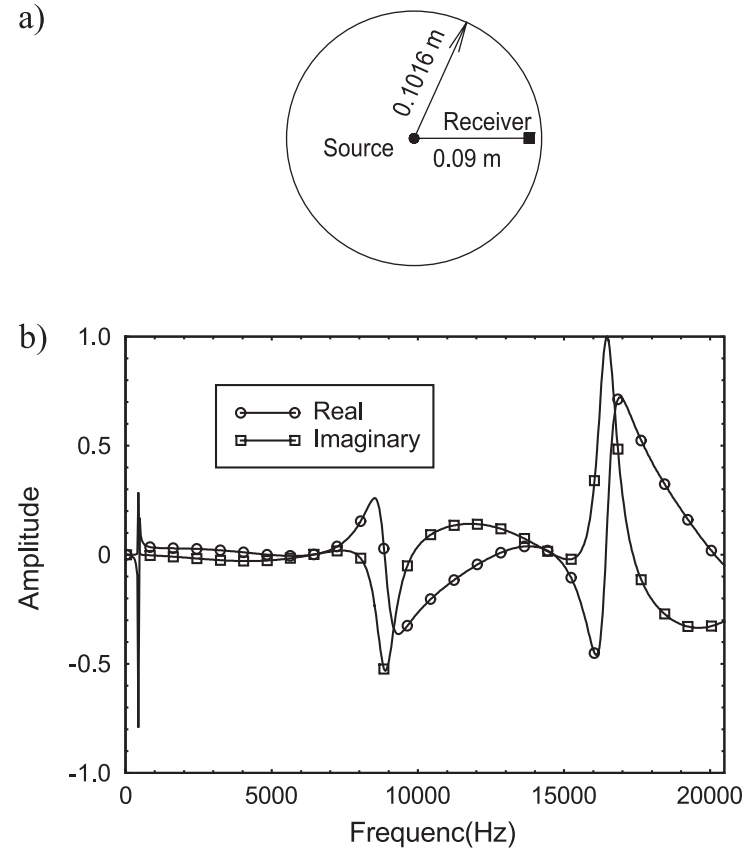

Fig. 1. Validation of the BEM algorithm: (a) geometry; (b) pressure response. In this figure, the solid line represents the analytical solution, and the marks illustrate the BEM solution.

\subsection{Fast formation}

In the simulations selected, the responses are computed in the frequency range $(40-20480 \mathrm{~Hz})$, with a frequency increment of $40 \mathrm{~Hz}$, defining the time signatures up to $T=1 / 40 \mathrm{~Hz}=25 \mathrm{~ms}$. The spatial distance between the virtual sources along the $z$ direction is set at $L=4 T \alpha=420.8 \mathrm{~m}$. The pressure time responses are obtained by modeling a spherical dilatational Ricker pulse source with a characteristic frequency of $6000 \mathrm{~Hz}$.

The number of boundary elements used to model each borehole varies with the excitation frequency. The ratio of the wavelength of the incident waves to the length of the boundary elements is greater than 28.0. A minimum of 120 boundary elements is used to discretize each borehole.

Figs. 4 and 5 display the results obtained at receiver lines $\mathrm{R} 1$ and $\mathrm{R} 2$, respectively, when the two boreholes are separated by 20.0 and the monopole source is excited close to the borehole wall. Both the time responses and their Fourier spectra representations are included, for a better visual distinction of the different wave types. The amplitude of the wavefield in the frequency vs. axial-wave number domain in fact allows easier recognition, identification, and physical interpretation of the different wave components, as they occupy specific sub-domains, according to their phase wave velocity.

Of the various waves excited by the source, two are non-dispersive body waves, namely, the dilatational (P) and shear (S) waves. The waves begin as dilatational waves in the fluid. As they reach the cylinder boundary, they are refracted into the formation as $\mathrm{P}$ or $\mathrm{S}$ waves, which are in turn refracted back into the fluid as $\mathrm{P}$ waves.

In addition, there are various types of guided waves - the normal modes - propagating along the interface between the fluid and solid: axisymmetric modes and modes with some azimuth variation. Certain modes, however, are excited only if the source excitation frequency exceeds the cutoff (or resonant) frequencies of the cylinder. The amplitude of these guided waves decays as they travel away from the borehole.

The position of these normal modes can be evaluated from the dispersion equation, which is derived by solving the wave equation for waves in the fluid and the solid and then by matching the boundary conditions at the fluid-solid interface. This yields a system of equations of the form $[\underline{\underline{K}}]$ $[\underline{X}]=[\underline{0}]$. The response is other than zero if the determinant $|\underline{\underline{K}}|$ is set to be zero. The solution of the resulting equation gives the required position of

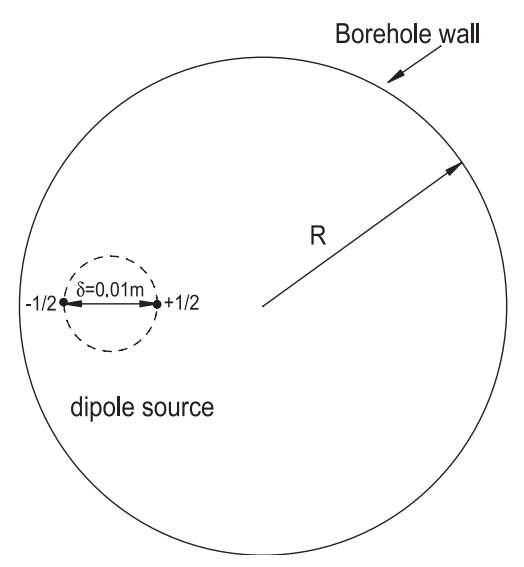

Fig. 2. Dipole sources. 
a)

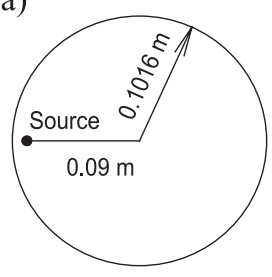

b)

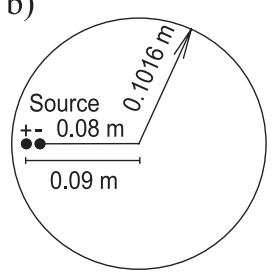

c)

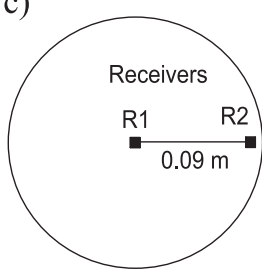

d)

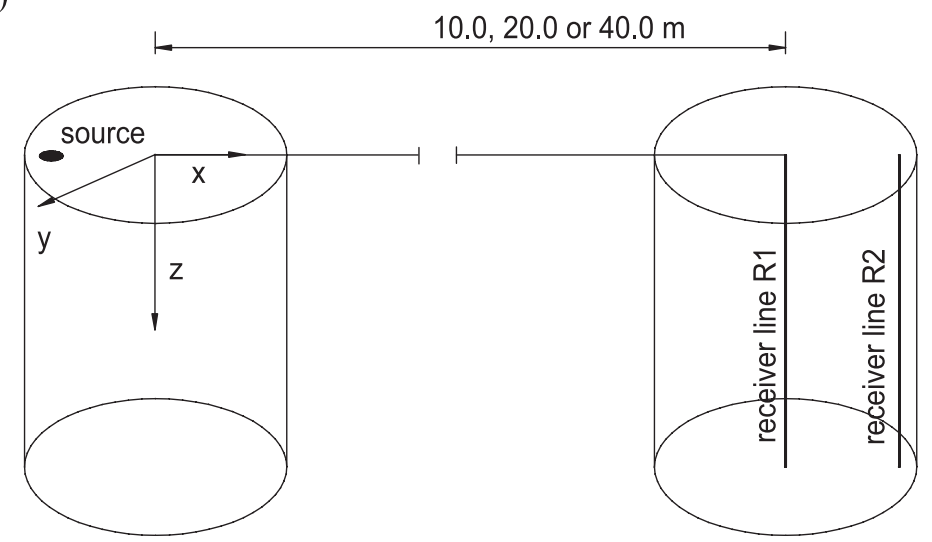

Fig. 3. Position of the sources and receivers: (a) monopole source; (b) dipole source; (c) receivers; (d) the geometry of the crosswell model, including the source location, the receiver locations, the distances between the wells and the orientation of the coordinate system.

these normal modes. All normal modes exhibit phase velocities lower than the $\mathrm{S}$ wave velocity of the formation.

As the non-dispersive (P) and shear (S) body waves reach the second borehole, they are refracted into the fluid of the second borehole, producing a complex wavefield that also involves the formation of additional guided waves.

The Fourier spectra are presented using a gray scale, ranging from white to black with increasing pressure amplitude. Lines associated with the $\mathrm{P}$ wave and $\mathrm{S}$ wave velocities of the formation, and the $\mathrm{P}$ wave velocity of the fluid have been included. These lines delineate the boundaries between normal modes, leaky modes and borehole resonances. Enhanced responses are visible for phase velocities larger than the $\mathrm{S}$ wave velocity, which corresponds to low values of the determinant $|\underline{\underline{K}}|$ but with an imaginary component. These modes are called leaky modes throughout this work. Their behavior follows the same trends as the pure normal modes, producing pressure oscillations within the borehole fluid, of the same type as the pure normal modes. The phase velocity does not represent the speed of energy transport, which is less than the shear wave velocity. The energy transport velocity or group velocities can be calculated numerically with the formula $U=d \omega / d k_{z}$.

The modes in the Fourier spectra are identified by a pair of numbers. The first number is the azimuth order, which indicates the variation of the mode with the azimuth, while the second is the radial order that supplies the variation of the mode with radial distance. Modes excited in the first borehole and with azimuthal variation may exhibit pressure values other than zero on the axis of the second borehole. However, their contribution is small when compared with the response generated by the excitation of the modes of the receiver borehole. The scale used in the plots does not allow all the modes excited to be identified easily, and this is particularly true of those with very low amplitudes. 

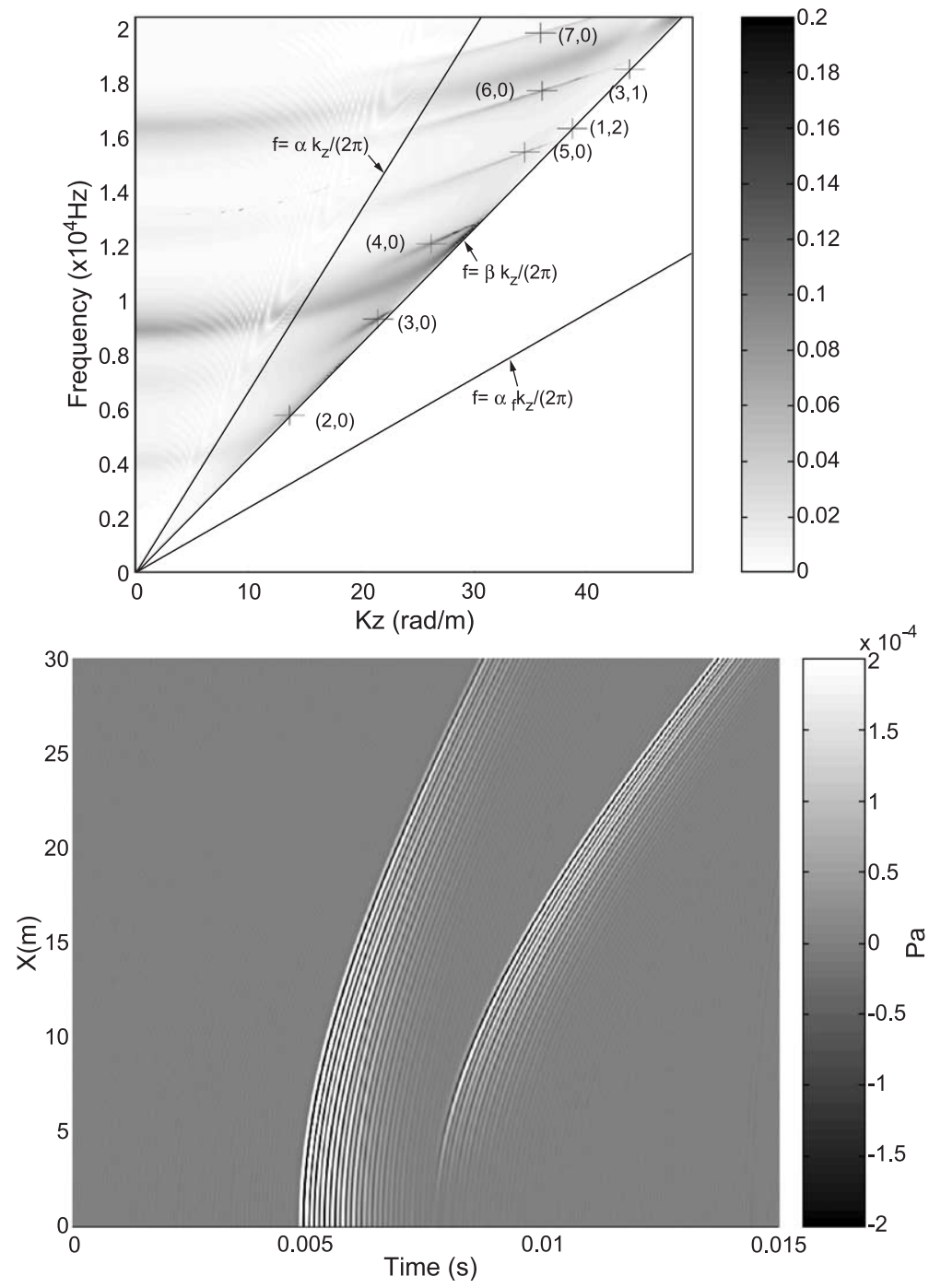

Fig. 4. Spectra and time responses when two boreholes, placed in a fast formation, $20.0 \mathrm{~m}$ apart, are excited by a monopole source placed close to the first borehole wall and registered at receiver line R1 located in the second borehole.

To allow an easier interpretation of the different modes excited, Fig. 6 plots the pressure computed over a fine grid of receivers placed inside the second borehole for frequency and axial wave number values corresponding to the points marked on the Fourier spectra results. The first axisymmetric mode would be the tube wave or Stoneley wave $(0,0)$. However, this mode is not visible in the second borehole. The first visible modes correspond to those with an azimuthal variation. Some of the modes with azimuthal variation are visible in the responses recorded at the $\mathrm{R} 1$ line of receivers (Fig. 4). These modes increase in importance for the R2 receivers (Fig. 5).

The computed time responses exhibit a set of strong pulses for waves traveling in the solid formation with $\mathrm{P}$ and $\mathrm{S}$ body wave velocities. These pulses are first generated by the interaction of the formation with $\mathrm{P}$ waves in the first borehole, they then travel through the elastic formation and eventually 

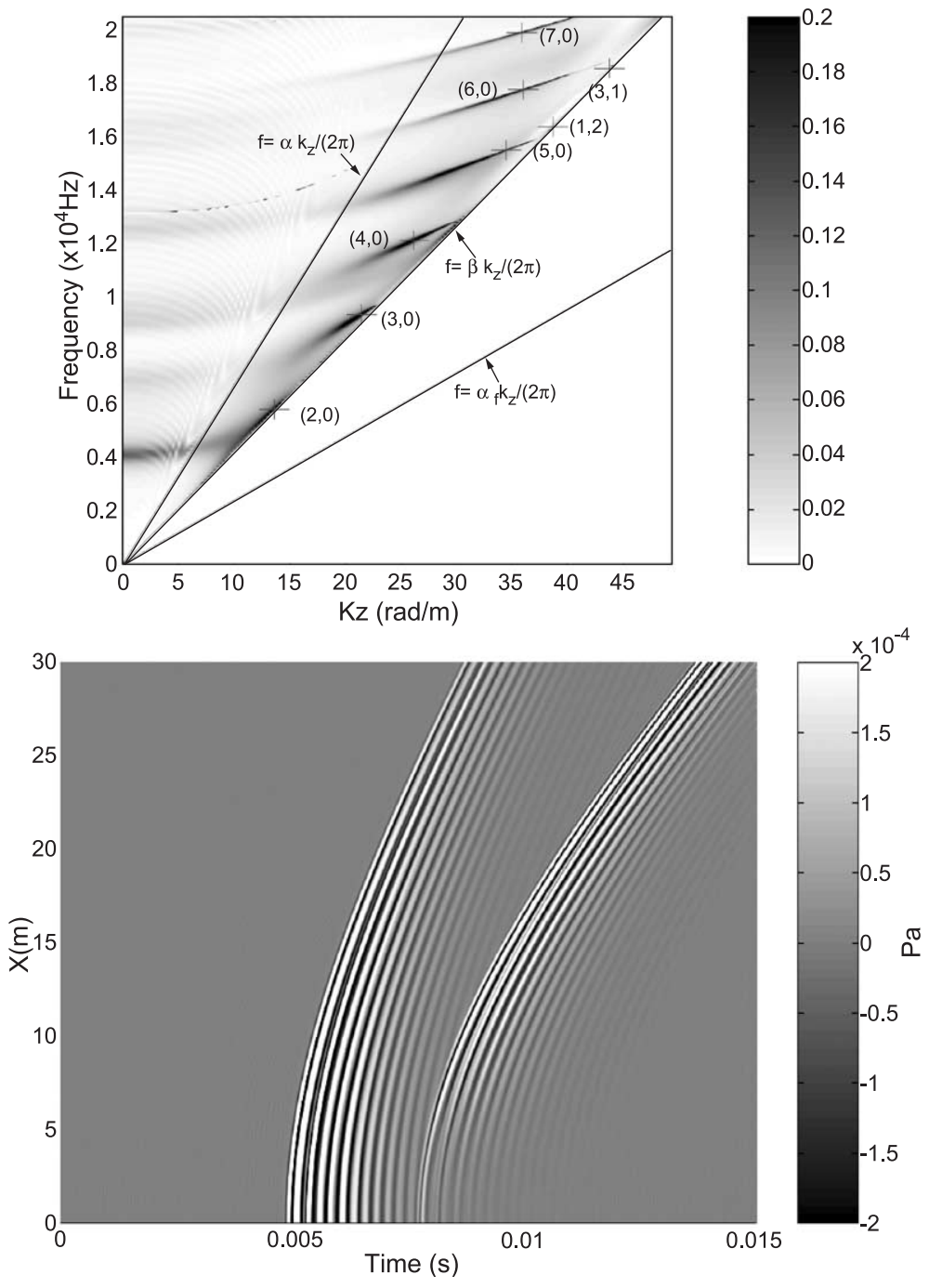

Fig. 5. Spectra and time responses when two boreholes, placed in a fast formation, $20.0 \mathrm{~m}$ apart, are excited by a monopole source placed close to the first borehole wall and registered at receiver line R2 located in the second borehole.

suffer the effect of multi-reflections within the second borehole. Analysis of the responses confirms that the importance of the $\mathrm{P}$ waves decreases whereas the contribution of the $\mathrm{S}$ waves grows as the distance between the receiver and the $z$ plane of the source increases.

Additionally, the analysis of the time plots shows that the responses for receivers placed in the vicinity of $z=0.0 \mathrm{~m}$ are not influenced by the presence of guided waves. As the receiver is placed farther away from $z=0.0 \mathrm{~m}$, the importance of these guided waves increases, and this is particularly noticeable for receivers placed in the vicinity of the borehole wall. The responses obtained at the receiver $z=30.0 \mathrm{~m}$ exhibit this behavior, indicating the arrival of waves traveling more slowly. The individual contribution of the various guided modes to the time responses is not easily distinguishable, given that the group velocities of the different modes are similar.

Analysis of the Fourier spectra responses confirms that the response decreases abruptly for axial 
$(1,0)$

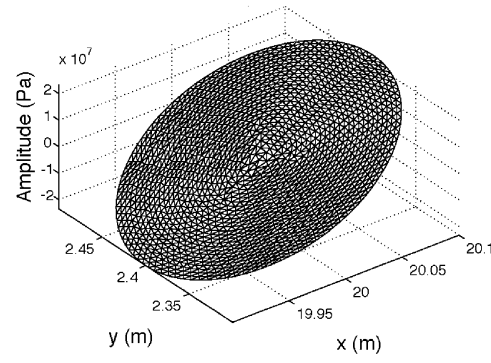

a)

$(4,0)$

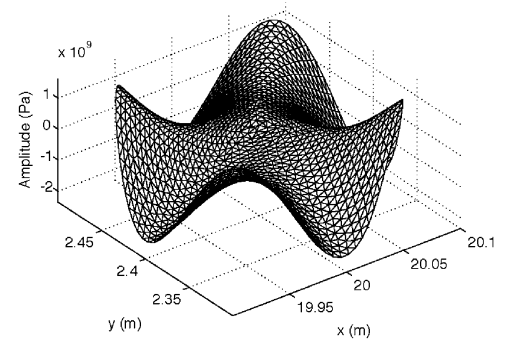

d)

$(6,0)$

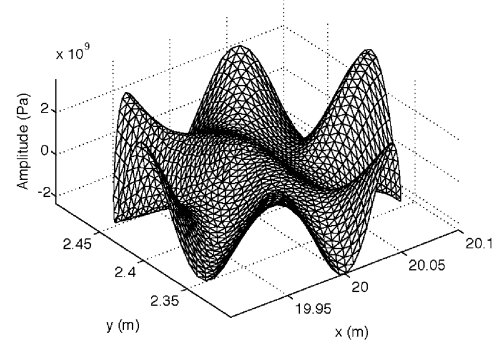

g)
$(2,0)$

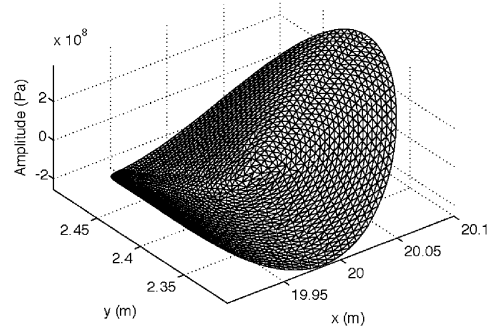

b)

$(5,0)$

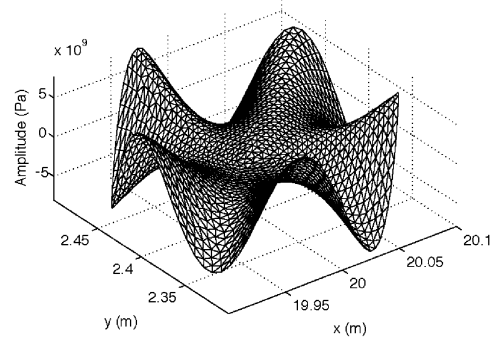

e)

$(3,1)$

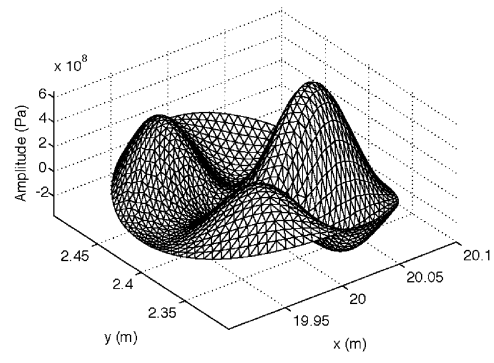

h)
$(3,0)$

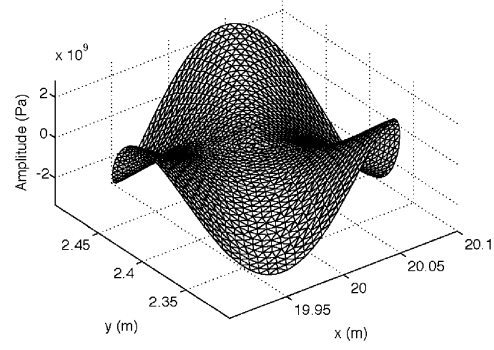

c)

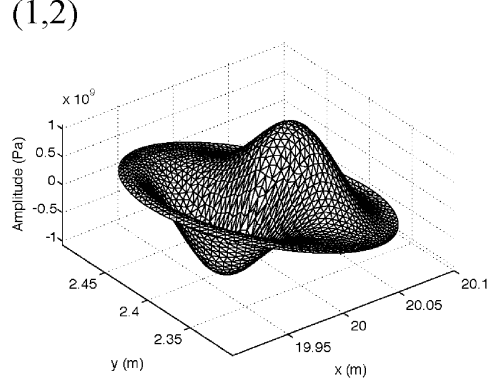

f)

$(7,0)$

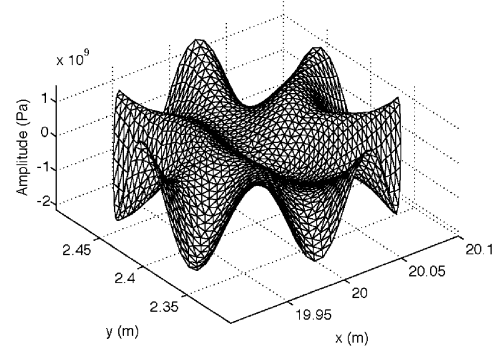

i)

Fig. 6. Pressure response over a fine grid of receivers placed inside the second borehole in a fast formation, $20.0 \mathrm{~m}$ from the first borehole, which contains a monopole source: (a) $f=3040.0 \mathrm{~Hz}, k_{z}=7.2 \mathrm{rad} / \mathrm{m}$; (b) $f=5784.0 \mathrm{~Hz}, k_{z}=13.5 \mathrm{rad} / \mathrm{m}$; (c) $f=9339.0 \mathrm{~Hz}, k_{z}=21.5 \mathrm{rad} / \mathrm{m}$; (d) $f=12126.0 \mathrm{~Hz}, k_{z}=26.1 \mathrm{rad} / \mathrm{m}$; (e) $f=15491.0 \mathrm{~Hz}, k_{z}=34.5 \mathrm{rad} / \mathrm{m}$; (f) $f=16375.0 \mathrm{~Hz}, k_{z}=38.6 \mathrm{rad} / \mathrm{m}$; (g) $f=17755.0 \mathrm{~Hz}, k_{z}=36.0 \mathrm{rad} / \mathrm{m}$; (h) $f=18560.0 \mathrm{~Hz}, k_{z}=43.7 \mathrm{rad} / \mathrm{m}$; (i) $f=19895.0 \mathrm{~Hz}, k_{z}=35.9 \mathrm{rad} / \mathrm{m}$.

wave numbers $\left(k_{z}\right)$ higher than those corresponding to $\omega / \beta$. The frequency domain results obtained at both receiver lines show the existence of different wave modes propagating in the dynamic system. These modes correspond to the guided and body waves generated by the excitation source. Some of these modes exhibit an azimuthal variation along the borehole cross-section and thus may not be recorded by specific receivers since they may be positioned in a nodal line (see Fig. 6).

Fig. 7 shows the response obtained at the two lines of receivers (R1 and R2) when the source excited is a dipole placed in the vicinity of the borehole wall. The responses evince features similar to those obtained in the presence of a monopole source. However, the amplitude of these responses 

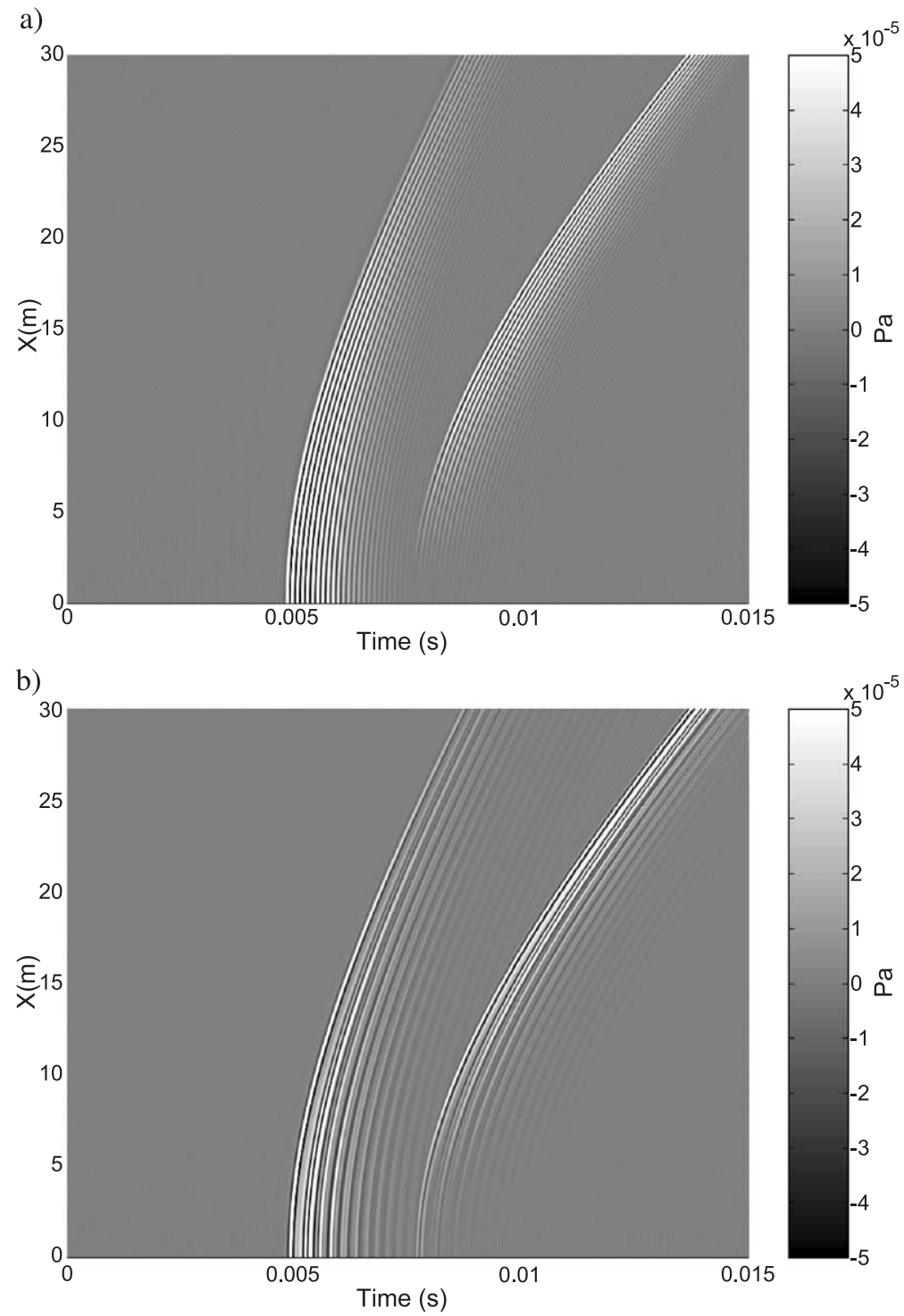

Fig. 7. Time responses when two boreholes, placed in a fast formation, $20.0 \mathrm{~m}$ apart, are excited by a dipole source placed close to the borehole wall: (a) receiver line $\mathrm{R} 1$; (b) receiver line $\mathrm{R} 2$.

decreases when the monopole is replaced by a dipole source.

The results presented in Fig. 8 correspond to those obtained at receiver line R2 when the boreholes are 10.0 and $40.0 \mathrm{~m}$ apart, in the presence of a monopole source placed close to the borehole wall. As expected, the amplitude of the responses is lower when the distance between the boreholes is greater. The responses display features similar to those observed when the boreholes are $20.0 \mathrm{~m}$ apart. However, the guided waves become more important as the distance between boreholes diminishes $(10.0 \mathrm{~m})$, while they lose importance when the distance is larger $(40.0 \mathrm{~m})$. Notice that when the boreholes are separated by a distance of $40.0 \mathrm{~m}$, the importance of the guided waves is slight, even for the receiver placed at $z=30.0 \mathrm{~m}$. 

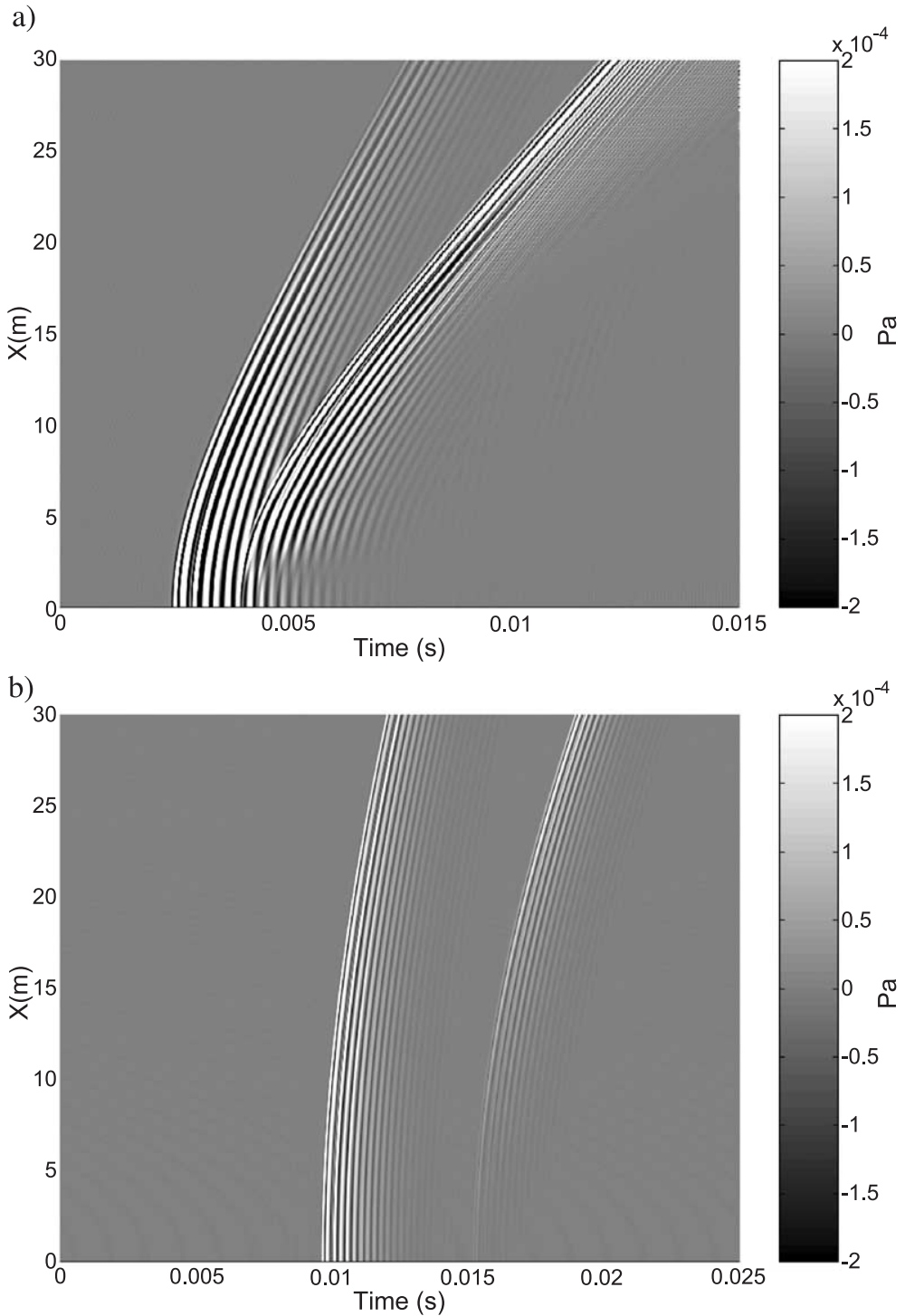

Fig. 8. Time responses at receiver line R2, when two boreholes, placed in a fast formation, are excited by a monopole source placed close to the borehole wall: (a) boreholes $10.0 \mathrm{~m}$ apart; (b) boreholes $40.0 \mathrm{~m}$ apart.

\subsection{Slow formation}

Figs. 9 and 10 display the time and Fourier spectra results obtained at receiver lines R1 and R2, respectively, when the two boreholes are $20.0 \mathrm{~m}$ apart and placed in a slow formation, and the monopole source is excited close to the borehole wall. The responses are calculated in the frequency range $(25 \mathrm{~Hz}, 12,800$ $\mathrm{Hz}$ ), with a frequency increment of $25 \mathrm{~Hz}$, defining time signatures up to $T=1 / 25=40 \mathrm{~ms}$. The frequency increment was changed in relation to the previous examples in the fast formation to allow a longer observation time given the slower velocity of the waves. The spatial distance between the virtual sources along the $z$ direction is set to $L=4 T \alpha=420.8$ $\mathrm{m}$. The source is modeled as a spherical dilatational Ricker pulse with a dominant frequency of $4000 \mathrm{~Hz}$. The simulation of a pulse with a higher characteristic 

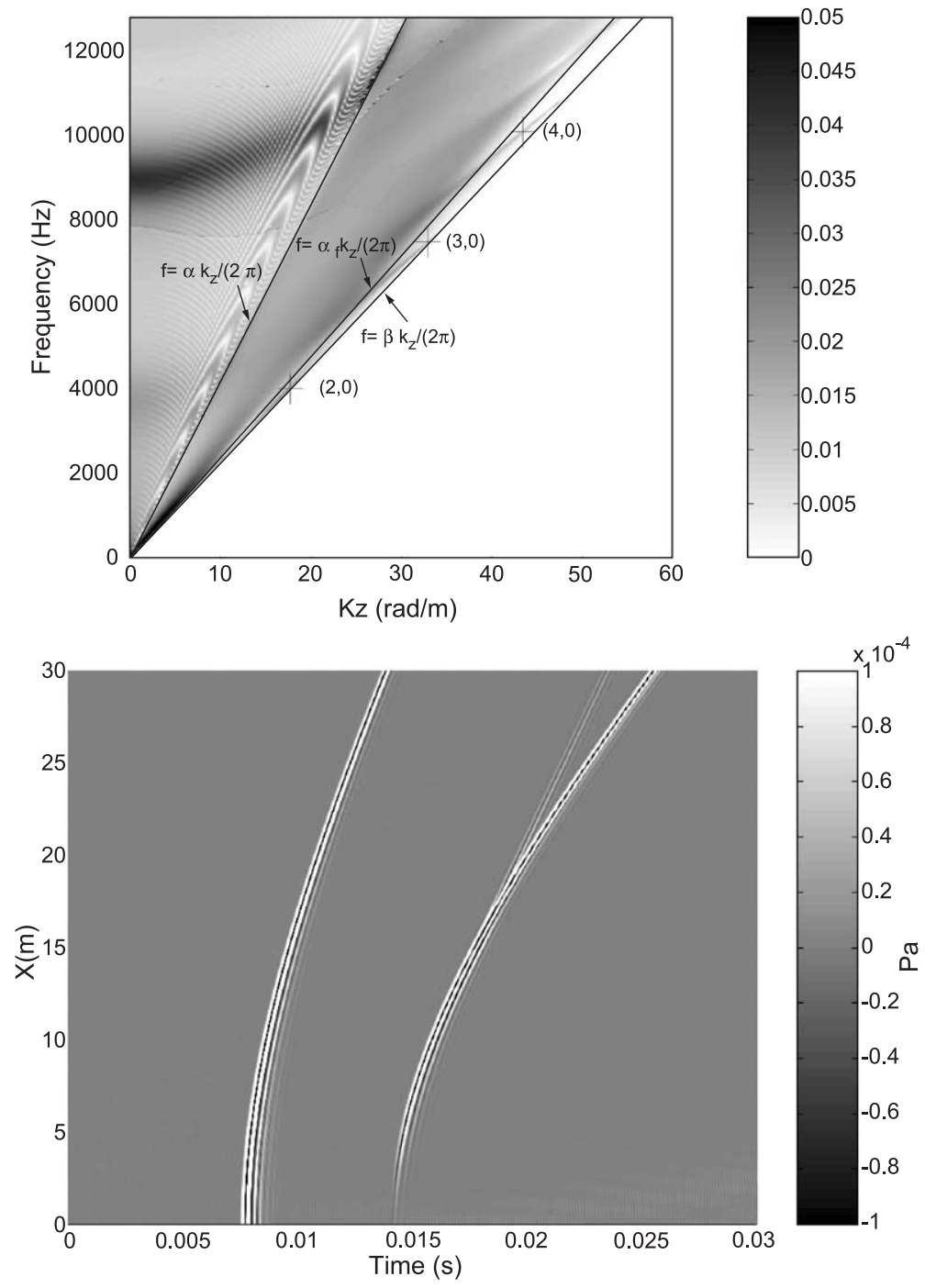

Fig. 9. Spectra and time responses, when two boreholes, placed in a slow formation, $20.0 \mathrm{~m}$ apart, are excited by a monopole source placed close to the first borehole wall, and registered at receiver line R1 located in the second borehole.

frequency would require the computation of additional frequencies.

The spectra plots allow the identification of different wave components, occupying specific sub-domains, according to their wave velocities. Again, it can be observed that the response decreases rapidly for axial wave numbers $\left(k_{z}\right)$ in excess of $\omega / \beta$. The modes in the Fourier spectra are again identified by a pair of numbers. Fig. 11 illustrates the pressure response over a fine grid of receivers placed inside the second borehole for the modes marked in the Fourier spectra plot. Again, there is no Stoneley wave $(0,0)$. Some of the modes with azimuthal variation are visible in the responses recorded at the R1 line of receivers, placed in the center of the borehole (Fig. 9). This is because the axis of the second borehole is not the axisymmetric axis of the dynamic system. However, these modes are more important for the R2 than the R1 line of receivers (Fig. 10).

The time plots clearly show the arrival of waves traveling with $\mathrm{P}$ and $\mathrm{S}$ body wave velocities in the 

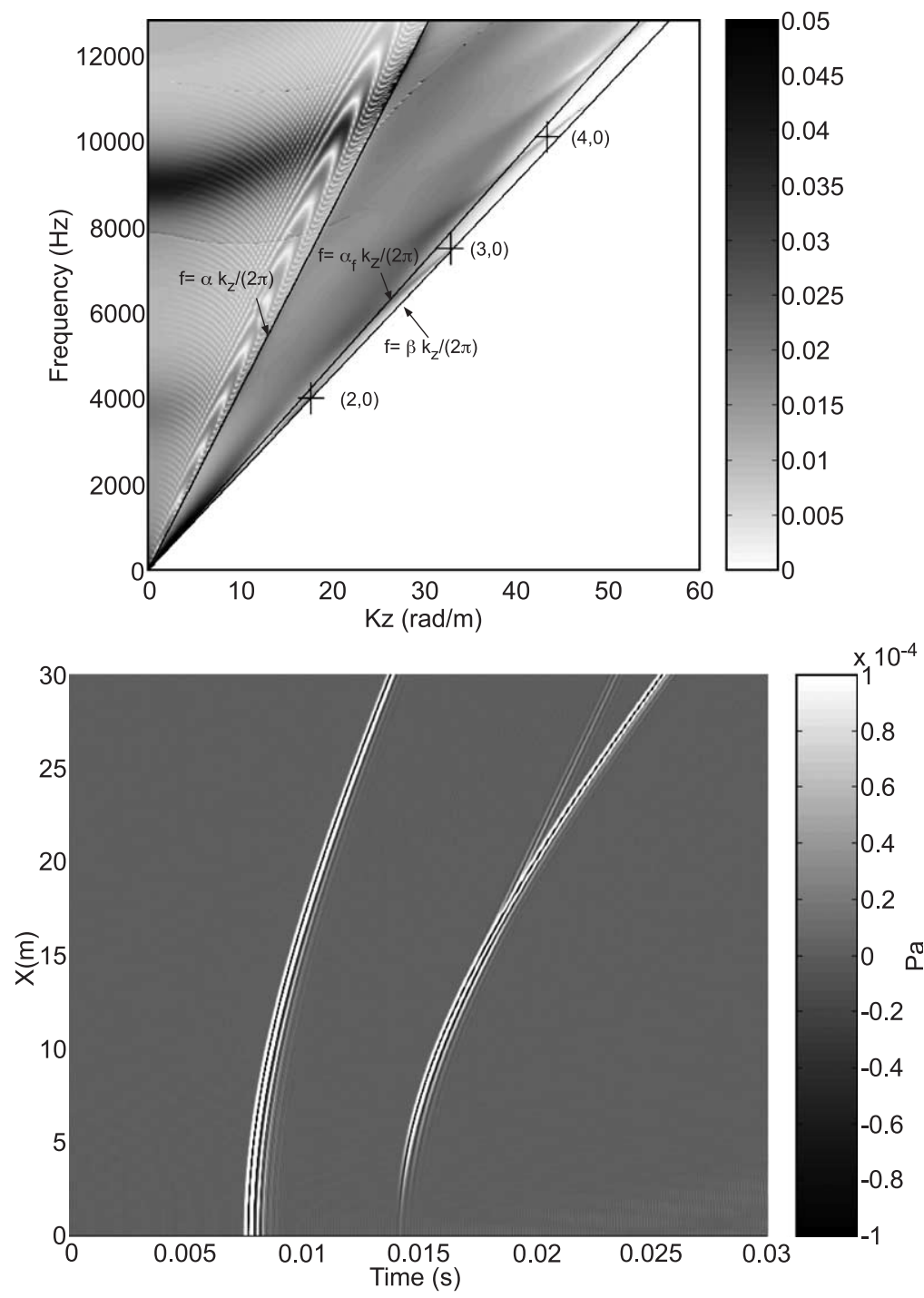

Fig. 10. Spectra and time responses, when two boreholes, placed in a slow formation, $20.0 \mathrm{~m}$ apart, are excited by a monopole source placed close to the first borehole wall, and registered at receiver line R2 located in the second borehole.

solid formation. An additional pulse is also visible, particularly for $z=30.0 \mathrm{~m}$. This mode can be identified as a result of the propagation of conical waves (Meredith et al., 1993; Samec, 1991, Rector and Hardage, 1992). This wave is the three-dimensional, axisymmetric space equivalent of a two-dimensional head-wave. As observed for the fast formation, the importance of the propagation of the guided waves along the borehole is only significant for receivers placed a long way from the $z$ plane of the source.
Again, it is hard to evaluate the contribution of each guided wave type from the time responses because they are associated with similar group velocities.

\section{Conclusions}

This work describes a boundary element method that was developed and implemented specifically to evaluate the 3D wave field generated by a dilatational 


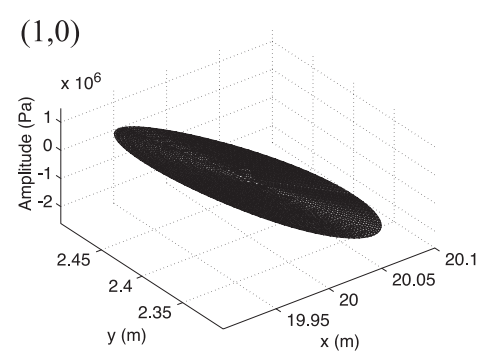

a)

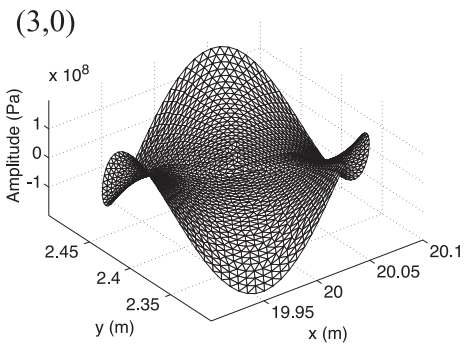

c)

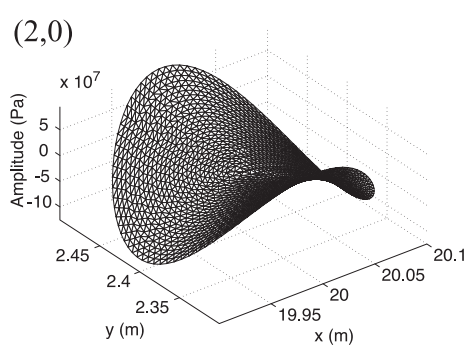

b)

$(4,0)$

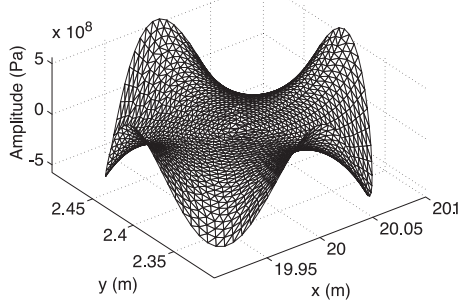

d)

Fig. 11. Pressure response over a fine grid of receivers placed inside the second borehole in a slow formation, $20.0 \mathrm{~m}$ from the first borehole, which contains a monopole source: (a) $f=1718.0 \mathrm{~Hz}, k_{z}=7.6 \mathrm{rad} / \mathrm{m}$; (b) $f=4000.0 \mathrm{~Hz}, k_{z}=17.7 \mathrm{rad} / \mathrm{m}$; (c) $f=7477.0 \mathrm{~Hz}, k_{z}=32.9 \mathrm{rad} / \mathrm{m}$; (d) $f=10082 \mathrm{~Hz}, k_{z}=43.5 \mathrm{rad} / \mathrm{m}$.

point load, in a simulation of the crosswell surveying technique.

The results given by the different simulations for slow and fast formations and for different borehole separations make it possible to conclude that the responses include signals from non-dispersive waves and waves produced by different modes. The Stoneley wave does not feature in the various computed responses because the distance separating the boreholes is too great. The contribution of the guided waves to the response is particularly important for large axial distances. It was difficult to separate the contribution to the response of each guided mode, since they exhibit similar group velocities.

When a dipole source is excited the results obtained have features similar to the ones yielded by a monopole source, besides the amplitude of the signals. It was found that the responses recorded in the presence of a dipole source have smaller amplitudes than those computed for a monopole source.

When the distance between the two boreholes increases, the amplitude of the response registered for both the monopole and the dipole sources falls.

\section{Acknowledgments}

Comments by the Editor in Chief, Jamie Rector, and two unnamed reviewers helped to improve the manuscript.

\section{References}

Albright, J.N., Johnson, P.A., 1990. Cross-borehole observation of mode conversion from borehole Stoneley waves to channel waves at a coal layer. Geophysical Prospecting 38, 607-620.

Ben-Menahem, A., Kostek, S., 1990. The equivalent force system of a monopole source in a fluid-filled open borehole. Geophysics 56, 1477-1481.

Beskos, D.E., 1997. Boundary Element Methods in Dynamic Analysis. part II: (1986-1996). Applied Mechanics Review 50, 149-197.

Bouchon, M., 1993. A numerical simulation of the acoustic and elastic wavefields radiated by a source in a fluid-filled borehole embedded in a layered medium. Geophysics 58, 475-481.

Bouchon, M., Aki, K., 1977. Discrete wave-number representation of seismic-source wave fields. Bulletin of the Seismological Society of America 67, 259-277.

Bouchon, M., Schmitt, D.P., 1989. Full wave acoustic logging in an irregular borehole. Geophysics 54, 758-765. 
Cheng, N., Cheng, C.H., Toksöz, M.N., 1995. Borehole wave propagation in three dimensions. Journal of the Acoustical Society of America 97, 3483-3493.

De Hoop, A.T., De Hon, B.P., Kurkjian, A.L., 1994. Calculation of transient tube wave signals in cross-borehole acoustics. Journal of the Acoustical Society of America 95, 1773-1789.

Dong, W., Bouchon, M., Toksöz, M.N., 1995. Borehole seismicsource radiation in layered isotropic and anisotropic media: boundary element modeling. Geophysics $60,735-747$.

Ellefsen, K.J., 1990. Elastic wave propagation along a borehole in an anisotropic medium. PhD, Department of Earth and Planetary Sciences, M.I.T.

Gibson, R.L., 1994. Radiation from seismic sources in cased and cemented boreholes. Geophysics 59, 518-533.

Gibson Jr., R.L., Peng, C., 1994. Low- and high-frequency radiation from seismic sources in cased boreholes. Geophysics $59,1780-1785$.

Kausel, E., 1992. Frequency domain analysis of undamped systems. Journal of Engineering Mechanics, ASCE 118, 721-734.

Krohn, C.E., 1992. Crosswell continuity logging using guided seismic waves. The Leading Edge 11, 39-45.

Kurkjian, A.L., Shu-Kong, C., 1986. Acoustic multipole sources in fluid-filled boreholes. Geophysics 51, 148-163.

Leslie, H.D., Randall, C.T., 1992. Multipole sources in boreholes penetrating anisotropic formations: numerical and experimental results. Journal of the Acoustical Society of America 91, 12-27.

Meredith, J.A., Toksöz, M.N., Cheng, C.H., 1993. Secondary shear waves from source boreholes. Geophysical prospecting 41, $287-312$.

Pao, Y.H., Mow, C.C., 1973. Diffraction of Elastic Waves and Dynamic Stress Concentrations, Crane and Russak, New York.

Peng, C., Toksöz, M.N., 1995. An optimal absorbing boundary condition for elastic wave modeling. Geophysics 60, 296-301.

Peng, C., Cheng, C.H., Toksöz, M.N., 1994. Cased borehole effects on downhole seismic measurements. Geophysical Prospecting 41, 883-912.

Peng, C., Lee, J.M., Toksöz, M.N., 1996. Pressure in a fluid-filled borehole caused by a seismic source in stratified media. Geophysics 61, 43-55.

Randall, C.T., 1991. Multipole acoustic waveforms in nonaxisymmetric boreholes and formations. Journal of the Acoustical Society of America 90, 1620-1631.
Rector, J.W., Hardage, B.A., 1992. Radiation pattern and seismic waves generated by a working roller-cone drill bit. Geophysics 57, 1319-1333.

Samec, P. V., 1991. Wave equation modeling: describing realistic media. PhD Thesis, Stanford University.

Stephen, R.A., Cardo-Casas, F., Cheng, C.H., 1985. Finite difference synthetic acoustic logs. Geophysics 50, 1588-1609.

Tadeu, A., Kausel, E., 2000. Green's functions for two-and-a-half dimensional elastodynamic problems. Journal of Engineering Mechanics, ASCE 126, $1093-1097$.

Tadeu, A., Santos, P., 2001. 3D Wave Propagation in Fluid-filled Irregular Boreholes in Elastic Formations. Journal of Soil Dynamics and Earthquake Engineering, 21, 499-517.

Tadeu, A., Santos, P.F.A., Kausel, E., 1999a. Closed-Form Integration of Singular Terms for Constant, Linear and Quadratic Boundary Elements: Part I. SH Wave Propagation. EABE-Engineering Analysis with Boundary Elements 23, 671-681.

Tadeu, A., Santos, P.F.A., Kausel, E., 1999b. Closed-form Integration of Singular Terms for Constant, Linear and Quadratic Boundary Elements: Part II. SV-P Wave Propagation. EABE-Engineering Analysis with Boundary Elements 23, $757-768$.

Tadeu, A., Godinho, L.M.C., António, J., 2001. Benchmark Solution for 3D Scattering from Cylindrical Inclusions. Journal of Computational Acoustics, 9, 1311-1328.

Tadeu, A., Godinho, L., Santos, P., 2002. Wave Motion Between Two Fluid Filled Boreholes in an Elastic Medium. Journal of EABE-Engineering Analysis with Boundary Elements, 26, $101-117$.

Toksöz, M.N., Cheng, C.H., Cicerone, R.D., 1992. Fracture detection and characterization from hydrophone vertical seismic profiling data. In: Evans, B., Wong, T. (Eds.), Fault Mechanics and Transport Properties of Rocks. Academic Press, New York, pp. 389-414.

White, J.E., Sengbush, R.L., 1963. Shear waves from explosive sources. Geophysics 28, 1001-1019.

Winbow, G.A., 1991. Seismic sources in open and cased boreholes. Geophysics 56, 1040-1050.

Yoon, K.H., McMechan, G.A., 1992. 3-D finite difference modelling of elastic waves in borehole environments. Geophysics 57, 793-804. 